\title{
Removing the spectral leakage in time-domain based near-field scanning measurements
}

\author{
Tim Claeys ${ }^{1}$, Dries Vanoost ${ }^{1,2}$, Joan Peuteman ${ }^{1,3}$, Guy A. E. Vandenbosch ${ }^{5}$, Fellow, IEEE \\ and Davy Pissoort ${ }^{1,4}$, Senior Member, IEEE \\ ${ }^{1}$ KU Leuven Technology Campus Ostend, ReMI Research Group, Ostend, Belgium \\ ${ }^{2}$ KU Leuven Kulak, Wave Propagation and Signal Processing Research Group, Kortrijk, Belgium \\ ${ }^{3}$ KU Leuven, Department of Electrical Engineering, Electrical Energy and Computer Architecture , Heverlee, Belgium \\ ${ }^{4}$ KU Leuven, Department of Electrical Engineering, Microelectronics and Sensors, Heverlee, Belgium \\ ${ }^{5}$ KU Leuven, Department of Electrical Engineering, TELEcommunications and MICrowaves, Heverlee, Belgium
}

\begin{abstract}
This paper describes the application of a new and easy to implement algorithm to EMI near-field scanning measurement results obtained with a time-domain based measurement system. The algorithm aims to reduce the effect of spectral leakage on amplitude and phase of the measured field components. The proposed algorithm significantly increases the accuracy of the measured electromagnetic near field with a limited extra computational cost. The versatility and effectiveness of the proposed algorithm is shown on both simulated and measured data. It is shown that for situations with one single frequency component or with several well-seperated frequency components, the algorithm is as performant as the application of a flat top window and outperforms other types of windows. However, as soon as the frequency components approach each other implying their spectral leakage patterns overlap, the proposed algorithm outperforms the application of a flat top window.
\end{abstract}

Index Terms-Near-Field scanning, Spectral Leakage, Discrete Fourier transform (DFT) and leakage effect, frequency domain, phase estimation.

\section{INTRODUCTION}

$\mathbf{C}$ URRENT electronic systems are constantly improving in both functionality and speed. At the same time, they are also squeezed into smaller volumes. Combination of both trends leads to several issues among which Electro-Magnetic Interference (EMI) is one of the major ones. To minimize the probability for EMI, appropriate design practices must be taken into account at every design step of the system. From a legal point of view, characterizing the radiated emission levels of the final electronic system is mandatory and is typically done in a (semi-) anechoic chamber, in a reverberation chamber or at an open area test site (OATS). These test environments require a significant investment and, therefore, are in many cases not directly available in the company itself. As a result, EMI measurements are postponed until the end of the design process. This implies an EMI problem will only be detected at the end of the design process when mitigating techniques are limited and expensive. Moreover, as the radiated emission is measured relatively far away from

Manuscript received XXX, XXXX. Corresponding author: Tim Claeys (email: tim.claeys@kuleuven.be)

Digital Object Identifier inserted by IEEE the device-under-test (D.U.T.), it is difficult to find the exact details of the cause of the EMI problem.

The advantages of a near-field scanner [1], [2] as a lowcost pre-certification and debugging test system have been seen by many before. The near field scanner measures the electromagnetic fields close to the device. Using such a near field scanner is much cheaper than the already mentioned traditional far field methods implying they can be used in every step of the design process. This approach allows to reduce the failure rate of the far field test methods performed after the last design step.

The near field scanning results can be used in several applications. Hotspots can be detected, the far-field can be estimated [3]-[5] and the currents at board level can be estimated [6], [7]. For hotspot detection, the absolute nearfield emission level is less important, finding the place of the hotspot is more important. When estimating the far-field or currents at board level, the absolute emission levels, both in magnitude and phase, are very important implying errors like spectral leakage (i.e. a frequency component not appearing at its frequency in the measured data and being spread over a frequency band) should not occur.

A common near field scanner consists of three main components: (i) a positioning system, (ii) a measurement probe and (iii) a measurement device. Three main measurement devices are frequently used, more precisely (i) a spectrum analyzer, (ii) a vector network analyzer (VNA) or (iii) an oscilloscope. When both magnitude and phase of the EM field are required, the most commonly used devices are a VNA or an oscilloscope [8]-[10]. A spectrum analyzer can also be used to measure the phase but requires several measurements at different heights [11] leading to longer measuring times. The main advantage of using an oscilloscope is the possibility to find intermittent problems, measuring several frequencies at once, thus reducing measurement time for several frequencies, and mapping time based magnitude plots [10]. While the use of an oscilloscope aims at reducing the measurement time for one spatial sampling point, complementary time reduction methods aim at minimizing the number of spatial sampling points. In [12]-[15] appropriate adaptive sampling algorithms are proposed to achieve this goal. 
A practical disadvantage of an oscilloscope is the huge amount of data that needs to be stored and transferred if every time-sample at every scanning point is stored. E.g., for a near-field scan of a relatively small PCB, having dimensions of $16 \mathrm{~cm}$ by $10 \mathrm{~cm}$, with a resolution of $1 \mathrm{~mm}$, the required data storage can easily exceed several tens of gigabytes. As measurements are done in the time domain, a Discrete Fourier Transform (DFT) or a Fast Fourier Transform (FFT) is needed to obtain the frequency spectrum of the measured signal directly after the time-domain sampling in order to reduce the data size (storing only the peak values). Unfortunately spectral leakage can heavily reduce the accuracy of the measured field values for a D.U.T. with small-band (i.e. clock based) emissions. As will be shown, spectral leakage can easily cause differences of $3.92 \mathrm{~dB}$ [16] in comparison with a Vector Network Analyzer (VNA) measurement.

Spectral leakage originates from a DFT, or its faster implementation the Fast Fourier Transform, when no coherent sampling is used. This means the sampled data does not contain exactly an integer number of periods of the original signal. However, realizing a coherent sampling procedure of the electromagnetic near field for a D.U.T. with a priori unknown frequency spectrum is very challenging. In principle, if the fundamental frequency of the radiating frequencies (e.g. due to a clock signal) is known, coherent sampling of this fundamental frequency and its harmonics can be obtained. However, if this fundamental frequency is not exactly known or if the fundamental frequency shifts in time due to heating or other reasons, it is a very difficult task to keep the sampling process coherent. Moreover, if the D.U.T. contains two radiating clock signals and the second clock frequency is not an integer multiple of the first clock frequency, it is not possible to avoid the spectral leakage phenomenon.

From literature it is known that by multiplying the original time signal with several kinds of windows, the effect of spectral leakage can be reduced [17]. These windows try to reduce the effect of spectral leakage by removing discontinuities and creating a smooth transient at the end and the beginning of the measured time interval. All window types have their advantages and disadvantages. While the use of one window is very good when estimating the amplitude of the signal, the other one will be good when estimating the frequency of the signal [16]. This paper applies a new and easy to implement algorithm to EMI NF scanning in order to reduce the effect of spectral leakage when combining the near field scanner with an oscilloscope. The algorithm gives a better estimation of the frequency, amplitude and phase of the measured electromagnetic field components. It will be shown that the algorithm gives much more accurate results in comparison with typical windows and this at a low computational cost. The algorithm performs very well when dealing with signals containing frequency components close to each other. Other approaches to suppress the spectral leakage already exist [18]-[22], but they do not rely on the sign changes further described in this paper.

The paper is organized as follows. In Section II the basic theory of the recently developed algorithm is summarized. Section III describes the measurement setup. In section IV the effect of spectral leakage is discussed on the spectrum of simulated time signals. Finally, section V evaluates the result when applying this algorithm on real life measured time signals. Section VI draws concluding remarks. In what follows, $\Re$ and $\Im$ are used for the real and imaginary part, respectively. The complex conjugate of $X$ is shown by using $\bar{X}$.

\section{ALGORITHM}

\section{A. The spectrum in case of spectral leakage}

Spectral leakage originates from a mismatch between the measuring time interval $\Delta T$ and an integer number of periods of the measured signal, introducing errors in the amplitude, phase and frequency readout. Spectral leakage occurs when calculating the spectrum of time continuous signals and time discrete i.e. sampled signals. The typical nature of this transformation error can be exploited to obtain an easy-to-implement algorithm to find the correct frequencies $f_{m}$, amplitudes $A_{m}$ and phases $\theta_{m}$ of the measured signal. In general, the measured time signal $x(l)$ consists of $M$ components whose frequencies are not related to each other. Every component in this time signal, which is not coherently sampled, suffers from the spectral leakage phenomenon, but also has a very typical pattern in its frequency spectrum around the component's exact frequency. Using this pattern, the correct frequency, amplitude and phase can be accurately estimated. Let us first consider a simple time signal (1) that only has one frequency component $f_{m}$. The measuring time interval $\Delta T$ contains $N$ samples with $l$ ranging from 0 to $N-1$.

$$
x(l)=A_{m} \cos \left(2 \pi f_{m} l \frac{\Delta T}{N}+\theta_{m}\right)
$$

The algorithm starts by applying a DFT (2) on the input signal (1). This results in a frequency spectrum $X(k)$ containing $\mathrm{N}$ components having order $k$. Let the order $a_{m}$ represent the correct order obtained from the correct frequency $f_{m}$ (3), representing the number of periods in the measured time interval. When $a_{m}$ is not an integer number, spectral leakage will occur.

$$
\begin{gathered}
X(k)=\frac{1}{N} \sum_{l=0}^{N-1} x(l) e^{-j 2 \pi k \frac{l}{N}} \\
a_{m}=f_{m} \Delta T
\end{gathered}
$$

Using (3), the input signal (1) can be rewritten as

$$
x(l)=\frac{A_{m}}{2} e^{j \theta_{m}} e^{j 2 \pi \frac{a_{m} l}{N}}+\frac{A_{m}}{2} e^{-j \theta_{m}} e^{-j 2 \pi \frac{a_{m} l}{N}} .
$$

The combination of (2) and (4), with $e^{j \pi \frac{a_{m}-k}{N}}$ as $\omega_{m}(k)$, can be written as

$$
\begin{aligned}
X(k)=\frac{1}{N} \sum_{l=0}^{N-1}\left(\frac{A_{m}}{2} e^{j \theta_{m}}\left(\omega_{m}(k)\right)^{2 l}\right. & \\
& \left.+\frac{A_{m}}{2} e^{-j \theta_{m}}\left(\overline{\omega_{m}(-k)}\right)^{2 l}\right) .
\end{aligned}
$$


If $X_{m}(k)$ is defined as

$$
\left.X_{m}(k)=\frac{1}{N} \sum_{l=0}^{N-1}\left(\frac{A_{m}}{2} e^{j \theta_{m}}\left(\omega_{m}(k)\right)^{2 l}\right)\right),
$$

(5) can be written as

$$
X(k)=X_{m}(k)+\overline{X_{m}(-k)} .
$$

Since for all complex numbers $\alpha \neq 1$, and for all integers $P_{2}$ and $P_{1}$ with $P_{2}>P_{1}$,

$$
\sum_{i=P_{1}}^{P_{2}} \alpha^{i}=\alpha^{P_{1}} \frac{1-\alpha^{P_{2}-P_{1}+1}}{1-\alpha},
$$

one can rewrite (6) as

$$
X_{m}(k)=\frac{A_{m} e^{j \theta_{m}}}{2 N} \frac{1-\left(\omega_{m}(k)\right)^{2 N}}{1-\left(\omega_{m}(k)\right)^{2}} .
$$

Using Euler and trigonometric functions, one obtains

$$
\begin{aligned}
1-\omega_{m}(k)^{2 N} & =-2 j \sin \left(\pi\left(a_{m}-k\right)\right) \omega_{m}(k)^{N} \\
1-\omega_{m}(k)^{2} & =-2 j \sin \left(\pi \frac{\left(a_{m}-k\right)}{N}\right) \omega_{m}(k) .
\end{aligned}
$$

The last factor in (9) can be rewritten using (10) and (11). In addition two assumptions will be made to rewrite (9) as (12). First $1-\frac{1}{N} \approx 1$ when $N$ is large. Second, $\sin \left(\pi \frac{a_{m}-k}{N}\right) \approx$ $\pi \frac{a_{m}-k}{N}$ when $\left(a_{m}-k\right)$ is significantly smaller than $N$ which is satisfied in the region of interest.

$$
X_{m}(k) \approx \frac{A_{m}}{2} \frac{\sin \left(\pi\left(a_{m}-k\right)\right)}{\pi\left(a_{m}-k\right)} e^{j\left(\pi\left(a_{m}-k\right)+\theta_{m}\right)}
$$

\section{B. Sign changes in a spectral leakage pattern}

The result from the DFT (7) contains two terms of the input signal (1) in the spectrum i.e. $X_{m}(k)$ for the positive frequencies and $\overline{X_{m}(-k)}$ for the negative frequencies. When the signal is coherently sampled ( $a_{m}$ is an integer), one can state that (12) equals $\frac{A_{m}}{2} e^{j \theta_{m}}$. When spectral leakage occurs $\left(a_{m}\right.$ is not an integer), the component $\frac{\sin \left(\pi\left(a_{m}-k\right)\right)}{\pi\left(a_{m}-k\right)}$ introduces several components surrounding the correct order $a_{m}$. Since all $k$ values are integer, $\sin \left(\pi\left(a_{m}-k\right)\right) e^{-j \pi k}=\sin \left(\pi a_{m}\right)$ and one can reduce (12) to

$$
X_{m}(k) \approx \frac{A_{m}}{2} \frac{\sin \left(\pi a_{m}\right)}{\pi\left(a_{m}-k\right)} e^{j\left(\pi a_{m}+\theta_{m}\right)} .
$$

When further investigating (13), it becomes clear that sign changes occur around and only around the correct order $a_{m}$. Table I shows these sign changes. In Table I $k_{m}=\left\lfloor a_{m}\right\rfloor$ and $k_{m}+1=\left\lceil a_{m}\right\rceil$.

These sign patterns occur in the real and in the imaginary part of $X_{m}(k)$. When $\pi\left(a-k_{m}\right)+\theta_{m}$ equals $\frac{\pi}{2}$ or $\frac{-\pi}{2}$, the real part of $X_{m}(k)$ equals zero and only the imaginary part of $X_{m}(k)$ can be used to determine $k_{m}$. When $\pi\left(a-k_{m}\right)+\theta_{m}$ equals 0 or $\pi$, the imaginary part of $X_{m}(k)$ equals zero and only the real part of $X_{m}(k)$ can be used to determine $k_{m}$.
TABLE I

SIGN PATTERN WHEN $k_{m}<a_{m}<k_{m}+1$

\begin{tabular}{c|c|c}
\hline$\gamma=\pi\left(a_{m}-k_{m}\right)+\theta_{m}$ & $k \leq k_{m}$ & $k \geq k_{m}+1$ \\
\hline \hline$\frac{-\pi}{2}<\gamma<\frac{\pi}{2}$ & $\Re\left(X_{m}(k)\right)>0$ & $\Re\left(X_{m}(k)\right)<0$ \\
\hline$\frac{\pi}{2}<\gamma<\frac{3 \pi}{2}$ & $\Re\left(X_{m}(k)\right)<0$ & $\Re\left(X_{m}(k)\right)>0$ \\
\hline $0<\gamma<\pi$ & $\Im\left(X_{m}(k)\right)>0$ & $\Im\left(X_{m}(k)\right)<0$ \\
\hline$\pi<\gamma<2 \pi$ & $\Im\left(X_{m}(k)\right)<0$ & $\Im\left(X_{m}(k)\right)>0$ \\
\hline
\end{tabular}

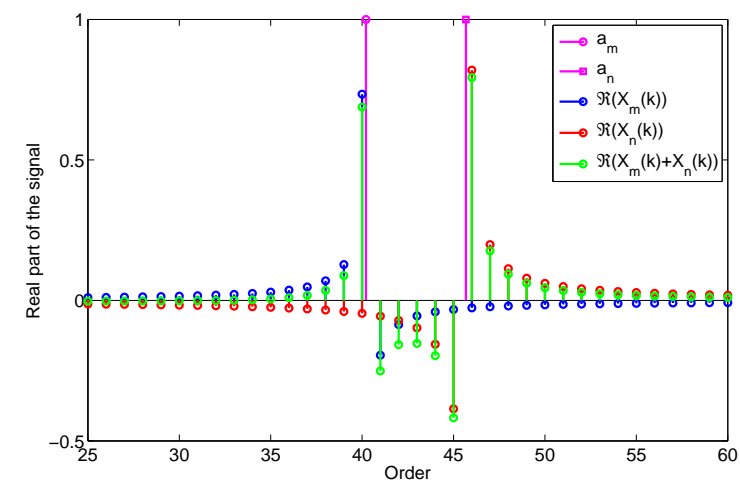

Fig. 1. Sign patterns $\Re\left(X_{m}(k)\right), \Re\left(X_{n}(k)\right)$ and $\Re\left(X_{m}(k)+X_{n}(k)\right)$

\section{Multiple frequencies}

When $M$ frequencies are considered in the input signal, the DFT output consists of $M$ spectral leakage patterns

$$
X(k)=\sum_{m=0}^{M}\left(X_{m}(k)+\overline{X_{m}(-k)}\right) .
$$

If the overlap of the the spectral groups of different sinusoidal components having a frequency $f_{n}$ (with $n \neq m ; n$ ranges from 1 to $M$ ) on the spectral group originating from $f_{m}$ is sufficiently small and the influence of the negative spectral group $\overline{X_{m}(-k)}$ on $X_{m}(k)$ is considered as negligible, it can be assumed that $X(k)=X_{m}(k)$ around $a_{m}$. The impact of the group originating from $f_{n}$ not only depends on the correct order $a_{n}$, the amplitude $A_{n}$ and the phase $\theta_{n}$ but also on $\left|a_{n}-a_{m}\right|$.

Figure 1 shows a simulation result when $a_{m}=40.21, a_{n}=$ 45.68, $A_{m}=A_{n}=2$ and $\theta_{m}=\theta_{n}=0$. Both correct orders $a_{m}, a_{n}$, their real parts $\Re\left(X_{m}(k)\right), \Re\left(X_{n}(k)\right)$ and the combined spectrum $\Re\left(X_{m}(k)+X_{n}(k)\right)=\Re(X(k))$ are shown. In order to use (15), the sign change between $\Re\left(X_{m}\left(k_{m}\right)\right)$ and $\Re\left(X_{m}\left(k_{m}+1\right)\right)$ is crucial. In order to obtain the same sign change between $\Re\left(X\left(k_{m}\right)\right)$ and $\Re\left(X\left(k_{m}+1\right)\right)$, $\left|\Re\left(X_{m}\left(k_{m}\right)\right)\right|$ and $\left|\Re\left(X_{m}\left(k_{m}+1\right)\right)\right|$, must be larger then $\left|\Re\left(X_{n}\left(k_{m}\right)\right)\right|$ and $\left|\Re\left(X_{n}\left(k_{m}+1\right)\right)\right|$. Once $k_{m}$ is known, using $\left|X\left(k_{m}\right)\right|$ and $\left|X\left(k_{m}+1\right)\right|$ in (15) instead of $\left|X_{m}\left(k_{m}\right)\right|$ and $\left|X_{m}\left(k_{m}+1\right)\right|$, not $a_{m}$ but an approximation $\hat{a}_{m}$ is obtained. Since $\left|a_{m}-\hat{a}_{m}\right| \leq 1$ and by (3) $a_{m}$ is proportional with $\Delta T$, the relative error on $a_{m}$ decreases by increasing $\Delta T$. Since $f_{m}=\frac{a_{m}}{\Delta T}$ and $\hat{f}_{m}=\frac{\hat{a}_{m}}{\Delta T}$, the relative error $\frac{\left(f_{m}-\hat{f}_{m}\right)}{f_{m}}$ also decreases.

Practical experience learns that $\left|a_{n}-a_{m}\right| \geq 5$ is usually sufficient in order to minimize the influences of the harmonic 
groups on each other, assuming that $\left|A_{n}\right|$ and $\left|A_{m}\right|$ are of the same order of magnitude. In addition $a_{n} \geq 3$ and $a_{m} \geq 3$ is required in order to minimize the influence of $\overline{X_{m}(-k)}$ on $X_{m}(k)$.

\section{Obtaining the correct order, amplitude and phase}

Once the region, $k_{m}$ and $k_{m}+1$ is known by looking for a sign change, a weighted average is taken from $k_{m}$ and $k_{m}+1$ in order to accurately determine the correct order $a_{m}$.

$$
a_{m} \approx \frac{\left|X\left(k_{m}\right)\right|\left(k_{m}\right)+\left|X\left(k_{m}+1\right)\right|\left(k_{m}+1\right)}{\left|X\left(k_{m}\right)\right|+\left|X\left(k_{m}+1\right)\right|}
$$

Using the correct order $a_{m}$ one can retrieve the amplitude and phase from (13). Equation (16) calculates a good approximation of the amplitude and equation (18) calculates a good approximation of the phase of the time signal. When $X\left(k_{m}+1\right)$ is larger than $X\left(k_{m}\right)$, better results can be achieved by using (17) instead of (16) and (19) instead of (18)

$$
\begin{gathered}
\frac{A_{m}}{2} \approx\left|X\left(k_{m}\right)\right| \frac{\pi\left(a_{m}-k_{m}\right)}{\left|\sin \left(\pi a_{m}\right)\right|} \\
\frac{A_{m}}{2} \approx\left|X\left(k_{m}+1\right)\right| \frac{\pi\left(k_{m}+1-a_{m}\right)}{\left|\sin \left(\pi a_{m}\right)\right|} \\
\theta_{m} \approx \Im\left(\ln \left(\frac{X\left(k_{m}\right)}{\frac{A_{m} \sin \left(\pi a_{m}\right)}{2 \pi\left(a_{m}-k_{m}\right)} e^{j \pi a_{m}}}\right)\right) \\
\theta_{m} \approx \Im\left(\ln \left(\frac{X\left(k_{m}+1\right)}{\frac{A_{m} \sin \left(\pi a_{m}\right)}{2 \pi\left(a_{m}-k_{m}-1\right)} e^{j \pi a_{m}}}\right)\right)
\end{gathered}
$$

\section{E. Practical approach}

The algorithm contains four main steps (Fig. 2). The first step calculates the DFT of the measured signal. The second step searches for sign changes in the spectrum (finding $k_{m}$ and $\left.k_{m}+1\right)$. Using $k_{m}$ and $k_{m}+1$ the correct order $a_{m}$ is calculated using (15). The fourth step calculates the amplitude (16) and phase (18).

When a frequency component is coherently sampled, the algorithm will not find a sign change in the spectrum for this specific frequency component. In order to ensure the frequency of every component will be found, the algorithm is executed twice on the same signal. During the first run all $N$ samples are used but during the second run only $N-1$ samples are used.

In the application of EMI near-field scanning (or in other related applications) the frequency components are not known in advance. This implies one can not ensure coherent sampling is obtained and there will be a large probability of having spectral leakage. In order to evaluate the algorithm in such applications, a case is presented. Ten sinusoidal components having random frequencies between $30 \mathrm{MHz}$ and $3 \mathrm{GHz}$ with random amplitudes and random phases are presented to the algorithm. First, a regular FFT and secondly a FFT in combination with different windows are compared with the algorithm. Fig. 3 shows the deviations on the amplitudes and Fig. 4 shows the deviations on the phases. It is clear the

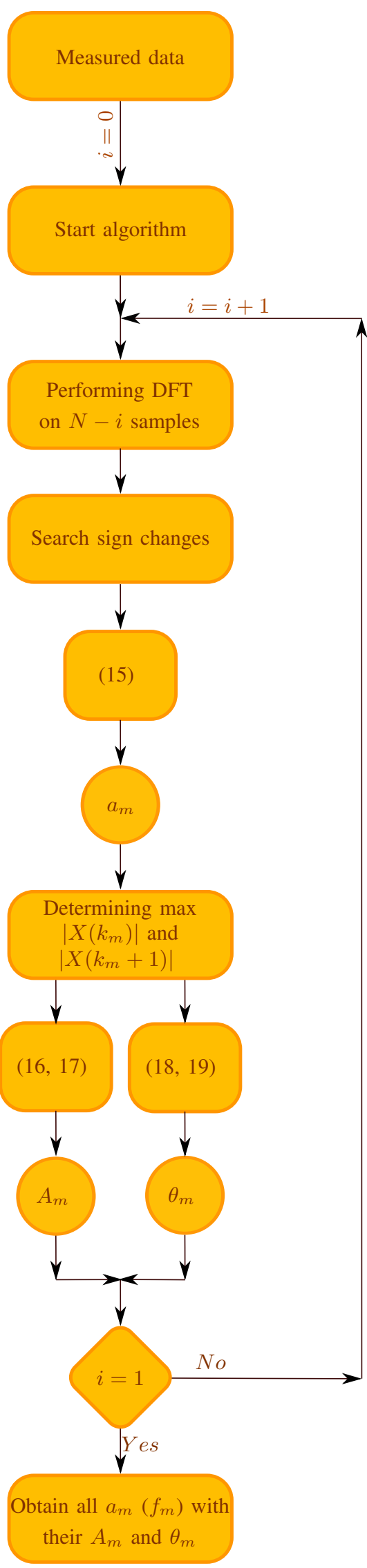

Fig. 2. Algorithm flowchart 


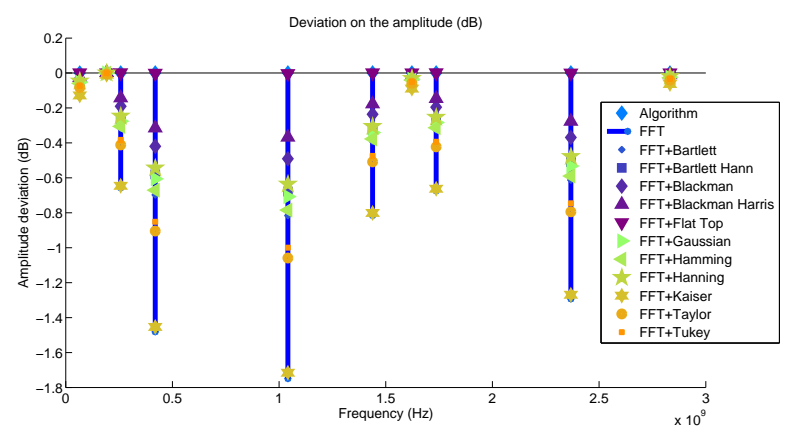

Fig. 3. Deviations on the amplitudes

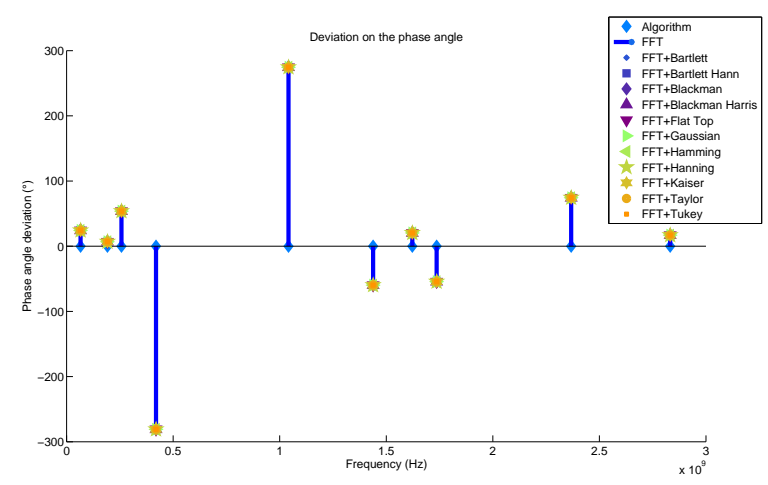

Fig. 4. Deviations on the phases

amplitudes obtained by the proposed algorithm are very close to the correct amplitudes. When using the FFT in combination with a flat top window, the obtained amplitudes are also close to the correct amplitude. In comparison with the regular FFT or a regular FFT in combination with other window types, the phase angles obtained by the algorithm are much closer to the correct phase angles. It should be noted that the phase deviations, when using different window shapes or the regular FFT, in figure 4 are close to each other, but not exactly the same.

\section{Measurement/Simulation SetuP}

\section{A. Near-field scanning system and probe}

Fig. 5 shows the near field scanning system available at the ReMI research group of KU Leuven Technology Campus Ostend. The system is built from a CNC milling machine. The miller and suspension were removed and replaced by a custom holder for a near-field probe. The near-field probe can be moved in three spatial dimensions above the D.U.T. The magnetic near-field probe is characterized for a frequency band of $30 \mathrm{MHz}$ to $3 \mathrm{GHz}$ and is produced by Langer EMVTechnik (RF-R 3-1). In order to measure the phase, a second probe is used as reference [9]. This second probe is a 3 $\mathrm{cm}$ loop magnetic near-field probe (7405-902B) from ETSLindgren's having a frequency range of $100 \mathrm{kHz}$ to $1.5 \mathrm{GHz}$. The maximum frequency of the measuring setup is hereby determined by the reference probe at $1.5 \mathrm{GHz}$. Each probe is connected to a $30 \mathrm{~dB}$ pre-amplifier manufactured by Langer

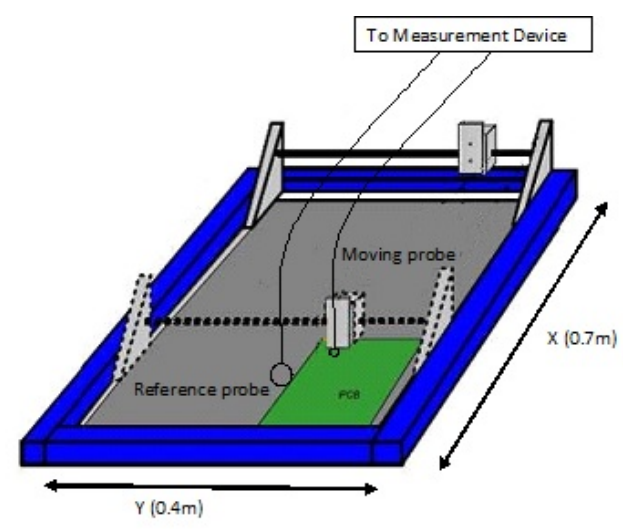

Fig. 5. Near-Field Scanner

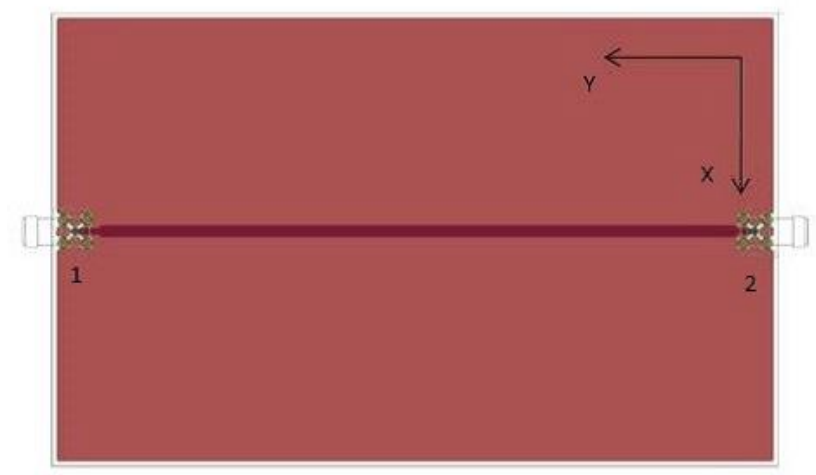

Fig. 6. PCB under test

EMV-Technik (PA303) and has a frequency range of $100 \mathrm{kHz}$ to $3 \mathrm{GHz}$.

\section{B. PCB under test}

In this paper, a Printed Circuit Board (PCB) (Fig. 6) is considered which contains a simple $50 \Omega$ microstrip on a 10 $\mathrm{cm}$ by $16 \mathrm{~cm}$ two-sided FR4 substrate having a thickness of $1.5 \mathrm{~mm}$.

\section{Simulations}

In order to show the effect of the spectral leakage phenomenon on a signal obtained by a near-field scan, a simulation was done using EMPRO [23] which is a full-wave electromagnetic solver. Two simulations are performed on the previously mentioned PCB (section III-B). The PCB is excited with a 1 volt $50 \Omega$ source (port 1 ) at $1 \mathrm{GHz}$ and terminated with a $50 \Omega$ impedance at port 2 . First the magnetic field $\left(H_{x}\right)$ is simulated in the frequency domain along a line over the $\mathrm{X}$ axis $(\mathrm{Y}=80 \mathrm{~mm})$ at a height of $5 \mathrm{~mm}$. In a second simulation the magnetic field is simulated via a point sensor at several places along the same line as the first simulation (201 places with an equidistance of $0.5 \mathrm{~mm}$ ). For each point sensor, the evolution of the magnetic field is simulated in the time-domain (sampled at $32.334 \mathrm{GHz}$ ) and the FFT is calculated. In Fig. 7 the measuring time of the point sensors equals an integer number of periods (coherent sampling) of the excitation signal 


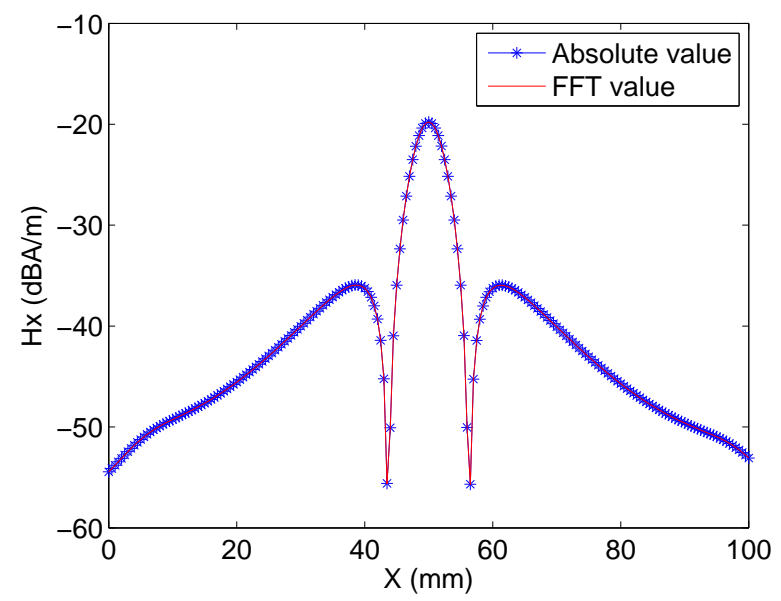

Fig. 7. Simulation without spectral leakage

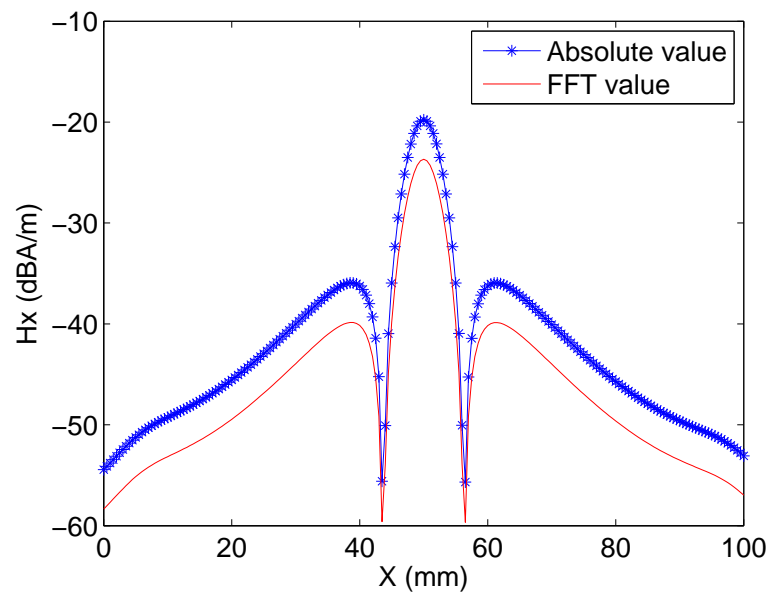

Fig. 8. Simulation with spectral leakage

avoiding spectral leakage. In Fig. 8, the measuring time equals an integer number of periods plus half of a period. It is clear the FFT suffers from spectral leakage which results in an error of approximately $3.92 \mathrm{~dB}$ as mentioned in [16].

In order to evaluate the algorithm, a comparison is made between the results when using a regular FFT, a regular FFT with different types of windows and finally the proposed algorithm. The data of Fig. 8 is used but instead of visualizing the entire line, only $\mathrm{X}=50 \mathrm{~mm}$ is considered. Table II shows

TABLE II

COMPARISON WINDOWS

\begin{tabular}{c|c||c|c}
\hline Window & Mag $(\mathrm{dBA} / \mathrm{m})$ & Window & Mag $(\mathrm{dBA} / \mathrm{m})$ \\
\hline \hline Exact & -19.92 & Gaussian & -21.3 \\
\hline Algorithm & -19.93 & Hamming & -21.45 \\
\hline FlatTop & -19.96 & Bartlett Hanning & -21.5 \\
\hline Blackman Harris & -20.65 & Tukey & -21.84 \\
\hline Blackman & -20.88 & Taylor & -21.97 \\
\hline Hanning & -21.16 & Kaiser & -23.27 \\
\hline Bartlett Hanning & -19.24 & FFT & -23.8 \\
\hline
\end{tabular}

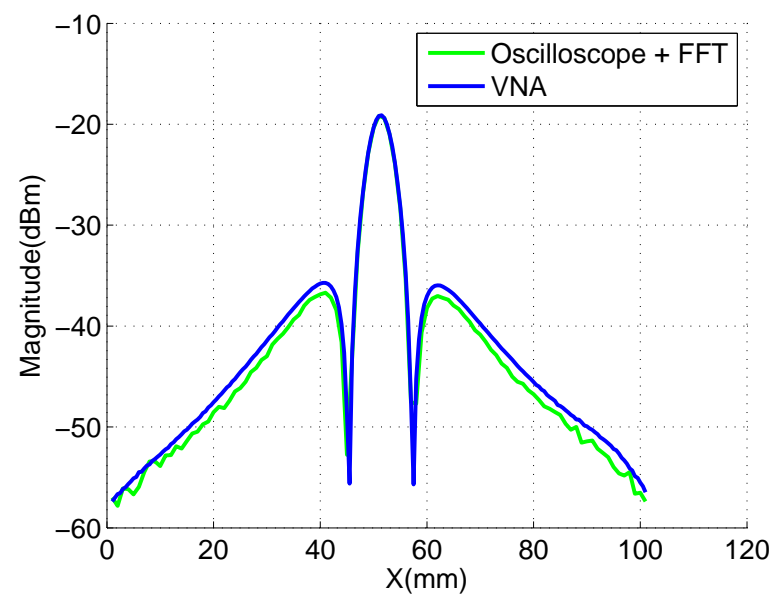

Fig. 9. FFT without spectral leakage, amplitude

the exact value (without spectral leakage), the results obtained by the algorithm, a regular FFT and several windows. It is clear that the algorithm, and the FFT in combination with the flat top window are closest to the correct value. The algorithm even outperforms the results obtained by the flat top window.

\section{Practical measurements}

\section{A. Amplitude}

This section mainly aims to obtain the same measurement results from an oscilloscope as from a VNA. The PCB presented in section III-B is excited with a $0 \mathrm{dBm} 1 \mathrm{GHz}$ signal. The same line as simulated in Section IV is now measured with an oscilloscope and a VNA. The oscilloscope has a $6.25 \mathrm{GHz}$ sampling rate and 100000 measurement points are saved which is a coherent sampling of the $1 \mathrm{GHz}$ signal (16000 periods). The amplitude result is presented in Fig. 9. In Fig. 9, no spectral leakage will occur since an integer number of periods is sampled. Suppose only 99997 measuring points are available, implying only 15999.52 periods, of the measured signal have been sampled and spectral leakage occurs. Fig. 10 shows that the difference has increased to $3.8 \mathrm{~dB}$. Other errors due to calibration errors of both the VNA and oscilloscope have been removed from these measurements. Simulations have shown that the algorithm provides better results than using the FFT in combination with a flat top window. But since the flat top approach also presents good results it is also considered in these measurement results. Fig. 11 shows the result when applying both approaches. Both the algorithm and the flat top window produce similar results. Having a closer look at Fig. 11, it is noticeable that both sidebands of the measured signal are not the same as the sidebands obtained by the Vector Network Analyzer approach. Indeed, the Signal to Noise Ratio (SNR) of the oscilloscope is much lower than the SNR of the VNA resulting in the corresponding error when small signals are being measured with the oscilloscope.

\section{B. Phase}

In near-field scanning the phase of the measured signal also needs to be measured when post-processing requires 


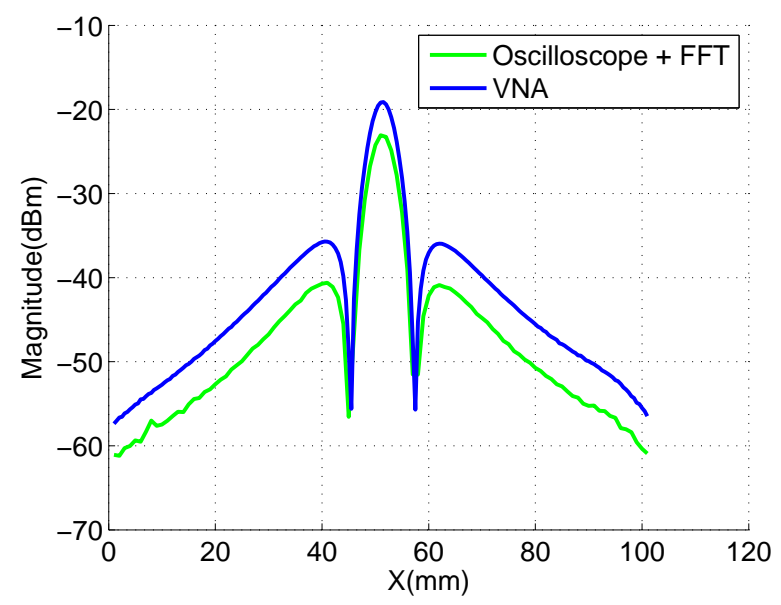

Fig. 10. FFT with spectral leakage, amplitude

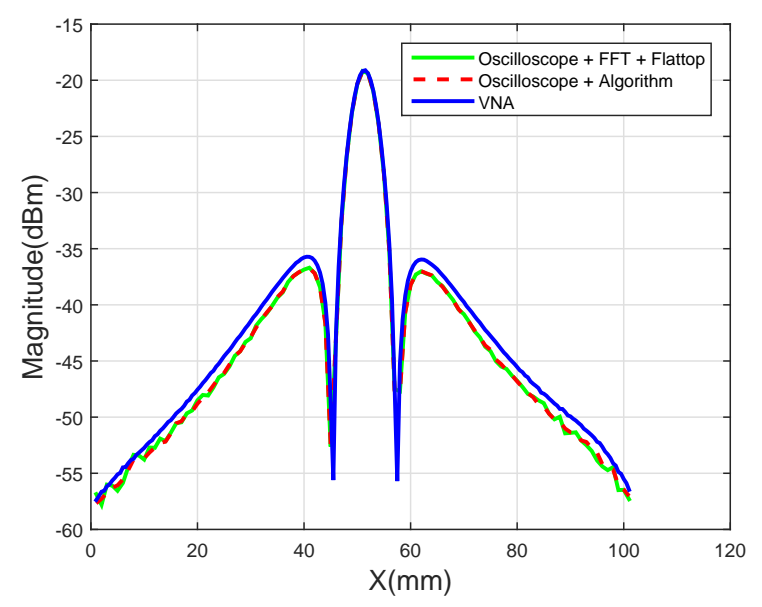

Fig. 11. Algorithm comparison, algorithm

the complex value of the measured near field. Hence the performance of the phase results of the algorithm also needs to be compared with a windowed FFT-approach (flat top). However, as phase-measurements require the use of two probes (one moving probe and one reference probe) [9], care has to be taken when applying the proposed algorithm. The frequencies determined by the moving and by the reference probe might be slightly different and slighty change from position to position. This will result in some added noise in these regions, as shown in Fig. 12. When the absolute magnitude and frequency of the reference probe is of limited importance, it is a better approach to apply the algorithm to the signal of the moving probe and to use the $a_{m}$ from the moving probe in the phase calculation of the reference probe. This modified algorithm results in much smoother results, as shown in Fig. 13.

\section{Performance on multiple signals}

It is not only important to evaluate the amplitude and phase, frequency resolution is also important in EMI near field scanning. Consider for example a switch mode power supply that switches at the rate of $200 \mathrm{kHz}$ and in its enclosure

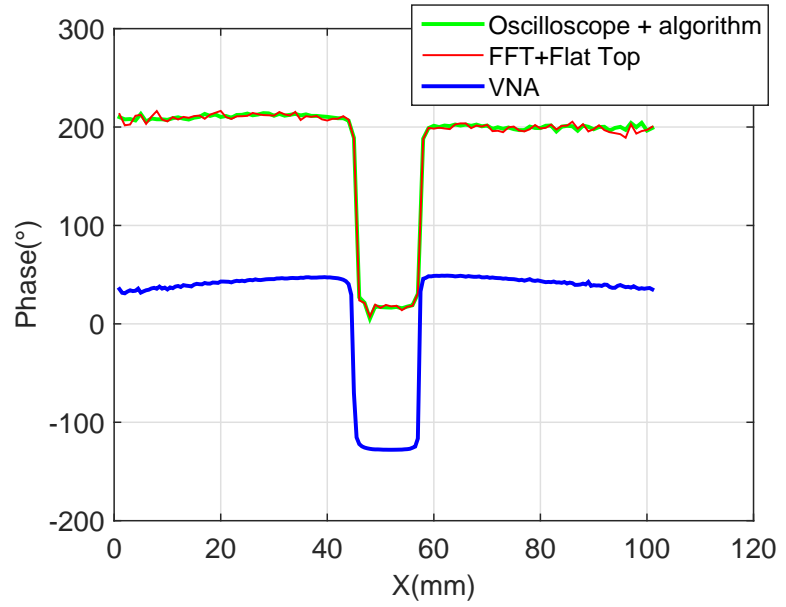

Fig. 12. Phase comparison, algorithm

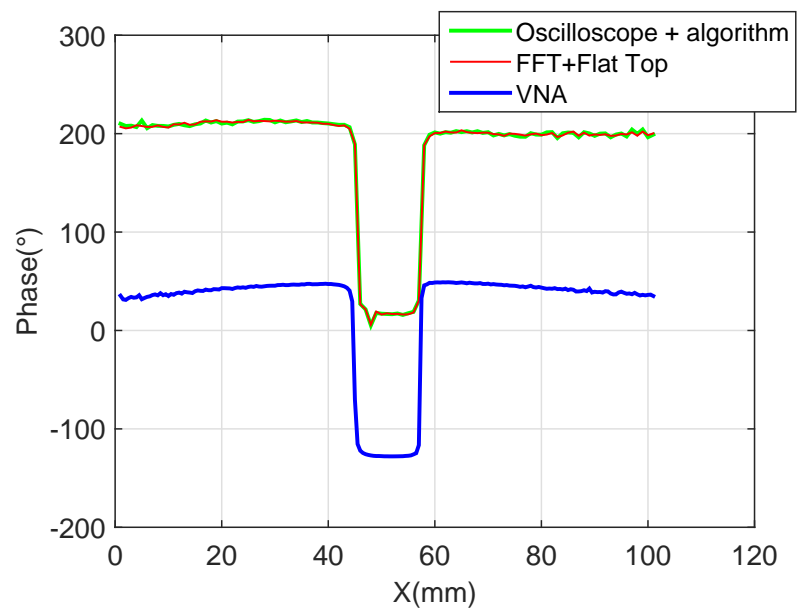

Fig. 13. Phase comparison, modified algorithm

radiates around $1 \mathrm{GHz}$. Multiple peaks will occur around $1 \mathrm{GHz}$ and the distance between the peaks will be $200 \mathrm{kHz}$. In order to simulate such a situation, the PCB in Section III-B is excited with two signals i.e. a signal at $1 \mathrm{GHz}$ and a signal at $999.8 \mathrm{MHz}$. The same test conditions are used in this setup as in the other measurement. When looking at one single point $(X=50)$ it is clear from Fig. 14 that there are two signals radiated by the PCB. The regular FFT shows both signals but with wrong amplitude results. The FFT + flat top window shows only one single radiating frequency. The algorithm shows two radiating frequencies and the error in comparison with the VNA measurement is small. The performance of the flat top window approach can be enhanced by measuring over a longer time, increasing the frequency resolution. But since a near field scan already takes a large amount of time, every increase of the measuring time has to be avoided.

\section{CONCLUSION}

This paper studies the use of a recently developed algorithm to reduce the error due to the spectral leakage phenomenon in time-domain based EMI NF measurements. The algorithm has 


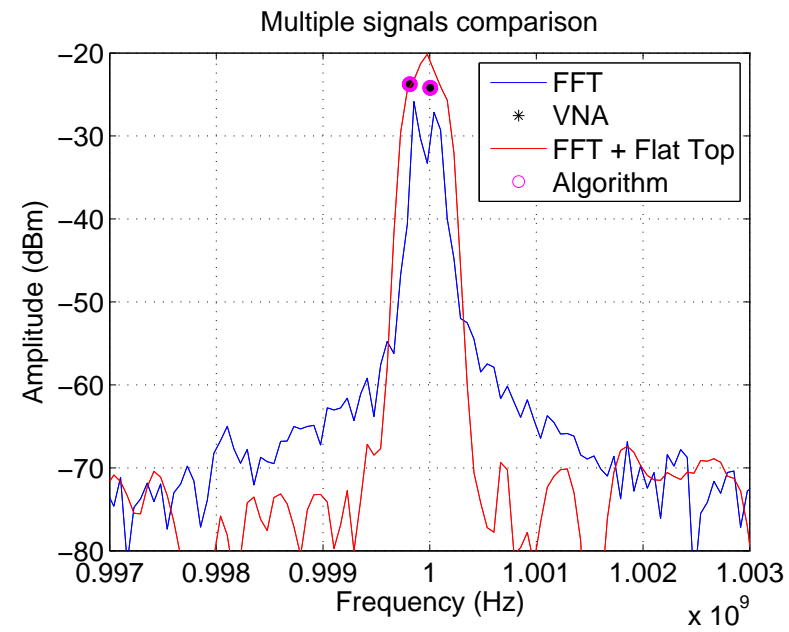

Fig. 14. Multiple signals

been used in a near-field scanner application. When simulating and measuring different situations that can occur in nearfield measurements, it becomes obvious that the proposed algorithm produces much better results than a regular FFT. The amplitude and phase results for signals with frequency components far enough from each other are comparable with the results of a flat top window. In order to distinguish two frequency components close to each other the algorithms performs remarkably better than the flat top window approach, which has a poor frequency resolution.

\section{ACKNOWLEDGMENT}

This research was supported by the IWT (Flanders, Belgium) under the TETRA-NEATH project. This doctoral research is funded by the Agency for Innovation by Science and Technology in Flanders (IWT).

\section{REFERENCES}

[1] K. Haelvoet, S. Criel, F. Dobbelaere, L. Martens, P. De Langhe, and R. De Smedt, "Near-field scanner for the accurate characterization of electromagnetic fields in the close vicinity of electronic devices and systems," in Instrumentation and Measurement Technology Conference, 1996. IMTC-96. Conference Proceedings. Quality Measurements: The Indispensable Bridge between Theory and Reality., IEEE, vol. 2, 1996, pp. 1119-1123 vol.2.

[2] D. Baudry, C. Arcambal, A. Louis, B. Mazari, and P. Eudeline, "Applications of the near-field techniques in emc investigations," Electromagnetic Compatibility, IEEE Transactions on, vol. 49, no. 3, pp. 485-493, Aug 2007.

[3] F. De Daran, J. Chollet-Ricard, F. Lafon, and O. Maurice, "Prediction of the field radiated at one meter from pcb's and microprocessors from near em field cartography," in Electromagnetic Compatibility, 2003. EMC '03. 2003 IEEE International Symposium on, vol. 1, May 2003, pp. 479-482 Vol.1.

[4] J.-S. Lee, T.-L. Song, J.-K. Du, and J.-G. Yook, "Near-field to far-field transformation based on stratton-chu fomula for emc measurements," in Antennas and Propagation Society International Symposium (APSURSI), 2013 IEEE, July 2013, pp. 606-607.

[5] V. Volski, G. A. E. Vandenbosch, and D. Pissoort, "Auxiliary Sources for the Near-to-Far-Field Transformation of Magnetic Near-Field Data," in Proceedings of the 2014 International Symposium on Electromagnetic Compatibility (EMC Europe 2014), vol. 2, no. 1, Gothenburg, Sweden, 2014, pp. 114-118.
[6] M. Hernando, A. Fernandez, M. Arias, M. Rodriguez, Y. Alvarez, and F. Las-Heras, "Emi radiated noise measurement system using the source reconstruction technique," Industrial Electronics, IEEE Transactions on, vol. 55, no. 9, pp. 3258-3265, Sept 2008.

[7] D. Rinas, S. Niedzwiedz, J. Jia, and S. Frei, "Optimization methods for equivalent source identification and electromagnetic model creation based on near-field measurements," in EMC Europe 2011 York, Sept 2011, pp. 298-303.

[8] X. Tong, "Simplified equivalent modelling of electromagnetic emissions from printed circuit boards," Ph.D. dissertation, University of Nottingham, 2010.

[9] T. Stadtler, L. Eifler, and J. ter Haseborg, "Double probe near field scanner, a new device for measurements in time domain," in Electromagnetic Compatibility, 2003 IEEE International Symposium on, vol. 1, Aug 2003, pp. 86-90 vol.1.

[10] T. Ordas, M. Lisart, E. Sicard, P. Maurine, and L. Torres, "Near-field mapping system to scan in time domain the magnetic emissions of integrated circuits," in Integrated Circuit and System Design. Power and Timing Modeling, Optimization and Simulation, ser. Lecture Notes in Computer Science, L. Svensson and J. Monteiro, Eds. Springer Berlin Heidelberg, 2009, vol. 5349, pp. 229-236. [Online]. Available: http : //dx.doi.org/10.1007/978 - 3-540-95948-92 3

[11] M. Johansson, H.-S. Lui, J.-C. Bolomey, and M. Persson, "Source modeling using phaseless low-frequency near-field measurements," Electromagnetic Compatibility, IEEE Transactions on, vol. 54, no. 3, pp. 613-624, June 2012.

[12] D. Deschrijver, F. Vanhee, D. Pissoort, and T. Dhaene, "Automated nearfield scanning algorithm for the EMC analysis of electronic devices," IEEE Transactions on Electromagnetic Compatibility, vol. 54, no. June 2012, pp. 502-510, 2012.

[13] B. Van der Streeck, F. Vanhee, B. Boesman, D. Pissoort, D. Deschrijver, I. Couckuyt, and T. Dhaene, "Practical implementation of a sequential sampling algorithm for EMI near-field scanning," in Proceedings of the 2012 International Symposium on Electromagnetic Compatibility (EMC Europe 2012). Rome, Italy: Ieee, Sep. 2012, pp. 1-5.

[14] T. Dorne, F. Vanhee, T. Grenson, D. Pissoort, D. Deschrijver, I. Couckuyt, and T. Dhaene, "Optimized sequential sampling algorithm for EMI near-field scanning," in Proceedings of the 2013 International Symposium on Electromagnetic Compatibility (EMC Europe 2013), Brugge, Belgium, 2013, pp. 385-388.

[15] T. Claeys, D. Pissoort, D. Deschrijver, I. Couckuyt, and T. Dhaene, "Sequential sampling algorithm for simultaneous near-field scanning of amplitude and phase," 2014 International Symposium on Electromagnetic Compatibility, pp. 79-84, Sep. 2014.

[16] F. Harris, "On the use of windows for harmonic analysis with the discrete fourier transform," Proceedings of the IEEE, vol. 66, no. 1, pp. 51-83, Jan 1978.

[17] F. Xu, "Algorithm to remove spectral leakage, close-in noise, and its application to converter test," in Instrumentation and Measurement Technology Conference, 2006. IMTC 2006. Proceedings of the IEEE, April 2006, pp. 1038-1042.

[18] M. Wu, D. Chen, and G. Chen, "New Spectral Leakage-Removing Method for Spectral Testing of Approximate Sinusoidal Signals," IEEE Transactions on Instrumentation and Measurement, vol. 61, no. 5, pp. 1296-1306, 2012.

[19] D. Agrez, "Weighted multipoint interpolated DFT to improve amplitude estimation of multifrequency signal," IEEE Transactions on Instrumentation and Measurement, vol. 51, no. 2, pp. 287-292, 2002.

[20] - "Spectrum analysis of waveform digitizers by IDFT and leakage minimization," in Proceedings of the Instrumentation and measurement technology Conference, 2005. IMTC 2005., 2005, pp. 17-19.

[21] F. Xu, "Algorithm to remove spectral leakage, close-in noise, and its application to converter test," in Proceedings of the Instrumentation and measurement technology Conference, 2006. IMTC 2006., 2006, pp. $1038-1042$.

[22] H.-C. Lin and C.-H. Chen, "Inter-harmonic identification using groupharmonic weighting approach based on the FFT," in Proceedings of the 33rd Annual Conference of the IEEE Industrial Electronics Society, Taipei, Taiwan, 2007.

[23] "Keysight empro," http://www.keysight.com, 2014. 


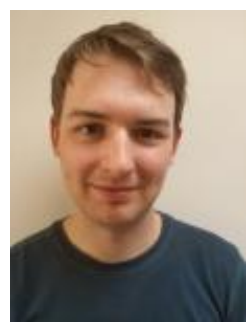

Tim Claeys was born in 1990. He received the M.S. industrial engineering sciences ,option electronics, from the University College Katholieke hogeschool Sint-Lieven Gent (KAHO Sint-Lieven), Ghent, Belgium, in 2013. He is currently working toward the Ph.D. degree in electrical engineering from the Katholieke Universiteit Leuven, Leuven, Belgium. Since 2013, he has been a Research Assistant at the ReMI research group (lab FMEC), KU Leuven Technology campus Ostend, which is a laboratory focusing on global reliability of electronic systems. His main research interests include near-field scanning and plane wave decomposition.

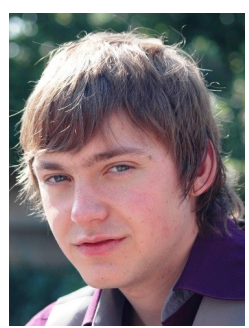

Dries Vanoost received the M.S. in engineering from the KU Leuven Technology campus Ostend in 2010. In 2012, he obtained a four year scholarschip from the Agency for Innovation by Science and Technology (IWT) of the Flemish government. Currently, he is working towards a $\mathrm{PhD}$ degree in electrical engineering. His research interests are electrical energy, electrical machines, actuators, magnetism and electromagnetic modeling.

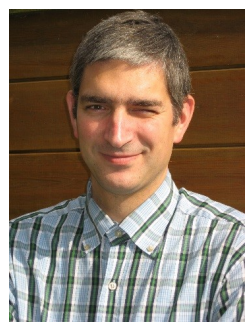

Joan Peuteman received the degree of industrial engineer in electrotechnics in 1990 from the Catholic University College of Bruges-Ostend, the M.S. degree in electrical engineering in 1993 and the $\mathrm{Ph}$. D. degree in 1999 from the University of Ghent, Belgium. He is currently teaching DSP and electrical engineering at the $\mathrm{KU}$ Leuven Technology Campus Ostend. His research interests include system theory, DSP, electromagnetism and power electronics.

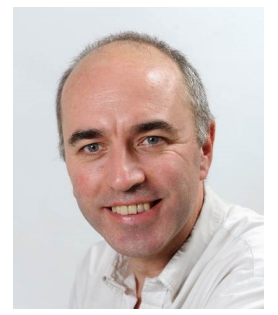

Guy A. E. Vandenbosch received the M.S. and $\mathrm{Ph} . \mathrm{D}$. degrees in Electrical Engineering from the Katholieke Universiteit Leuven, Leuven, Belgium, in 1985 and 1991, respectively. From 1991 to 1993, he held a postdoctoral research position at the Katholieke Universiteit Leuven. Since 1993, he has been a Lecturer, and since 2005, a Full Professor at the same university. Guy Vandenbosch has teached or teaches courses on "Electromagnetic Waves", "Antennas", "Electromagnetic Compatibility", "Electrical Engineering, Electronics, and Electrical Energy", and "Digital Steer- and Measuring Techniques in Physics". His research interests are in the area of electromagnetic theory, computational electromagnetics, planar antennas and circuits, nano-electromagnetics, EM radiation, EMC, and bio-electromagnetics. His work has been published in ca. 200 papers in international journals and has been presented in ca. 300 papers at international conferences. Guy Vandenbosch has been a member of the "Management Committees" of the consecutive European COST actions on antennas since 1993. Within the ACE Network of Excellence of the EU (2004-2007), he was a member of the Executive Board and coordinated the activity on the creation of a European antenna software platform. At present, he leads the EuRAAP Working Group on Software and represents this group within the EuRAAP Delegate Assembly. From 2001 to 2007, he was the President of SITEL, the Belgian Society of Engineers in Telecommunication and Electronics. Since 2008, he is a member of the board of FITCE Belgium, the Belgian branch of the Federation of Telecommunications Engineers of the European Union. In the period 1999-2004, he was vice-chairman, and in the period 2005-2009 secretary of the IEEE Benelux Chapter on Antennas en Propagation. Currently he holds the position of chairman of this Chapter. In the period 2002-2004 he was secretary of the IEEE Benelux Chapter on EMC. He currently is secretary of the Belgian National Committee for Radio-electricity (URSI), where he is also in charge of commission E. Guy Vandenbosch is a fellow of the IEEE.

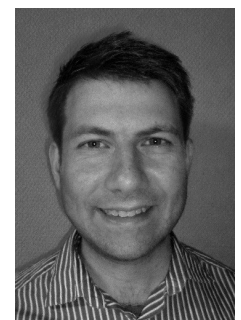

Davy Pissoort was born in 1978. He received the M.S. and Ph.D. degrees in electrical engineering from Ghent University, Ghent, Belgium, in 2001 and 2005, respectively. From October 2005 to October 2006, he was a Postdoctoral Researcher Ghent University, Belgium. From November 2006 to July 2009, he was a Research Engineer at the EesofEDA Department of Agilent Technolgies in Belgium. Since August 2009, he is Assistant Professor at KU Leuven, Campus Ostend, Belgium where he is also head of the research group Reliability in Mechatronics and ICT. His current research interests include the development of fast and efficiënt electromagnetic modelling methods for EMC, SI and PI, the development of characterization methods for shielding materials and gaskets, as well as the analysis and testing of the mechanical and thermal reliability of electronic modules. He is Senior Member of the IEEE EMC Society and member of the International Steering Committee of EMC Europe. In September 2013 he was vice-chair for the EMC Europe 2013 Symposium in Brugge, Belgium. 